\title{
On the convergence of a high-accuracy compact conservative scheme for the modified regularized long-wave equation
}

\author{
Xintian $\operatorname{Pan}^{1,2^{*}}$ and Luming Zhang ${ }^{3}$
}

${ }^{*}$ Correspondence:
panxintian@126.com
${ }^{1}$ School of Mathematics
and Information Science,
Weifang University,
Weifang 261061, China
Full list of author information
is available at the end of the
article

${ }^{*}$ Correspondence: panxintian@126.com and Information Science, Weifang University, Full list of author information article

\begin{abstract}
In this article, we develop a high-order efficient numerical scheme to solve the initialboundary problem of the MRLW equation. The method is based on a combination between the requirement to have a discrete counterpart of the conservation of the physical "energy" of the system and finite difference method. The scheme consists of a fourth-order compact finite difference approximation in space and a version of the leap-frog scheme in time. The unique solvability of numerical solutions is shown. A priori estimate and fourth-order convergence of the finite difference approximate solution are discussed by using discrete energy method and some techniques of matrix theory. Numerical results are given to show the validity and the accuracy of the proposed method.
\end{abstract}

Keywords: The MRLW equation, Compact conservative method, Solvability, Convergence, Stability

\section{Background}

The generalized regularized long-wave (GRLW) equation reads (Peregrine 1996):

$$
u_{t}-\mu u_{x x t}+u_{x}+\alpha u^{p} u_{x}=0,
$$

where $\alpha$ and $\mu$ are positive constants, $p \geq 1$ is an integer. When $p=1$, the Eq. (1) is usually called as the regularized long-wave (RLW) equation proposed by Peregrine (1996) and Benjamin et al. (1972) to describe nonlinear dispersive waves. Various numerical techniques have been developed to solve this equation. These partly include finite difference method, finite element methods, least squares method and collocation method with quadratic B-splines, cubic B-splines and septic splines, multisymplectic numerical method, in this respect, we refer readers to Kutluay and Esen (2006), Zhang (2005), Avilez-Valente and Seabra-Santos (2004), Esen and Kutluay (2006), Guo and Chen (2006); Gu and Chen 2008), Saka and Dag (2008), Dag (2000), Dag and Ozer (2001), Dag et al. (2004), Soliman and Raslan (2001), Soliman and Hussien (2005), Cai (2009, 2011), and references therein. Another special case of Eq. (1) for $p=2$, the modified regularized long-wave (MRLW) equation is given by

$$
u_{t}-\mu u_{x x t}+u_{x}+\alpha u^{2} u_{x}=0 .
$$


In recent years, the MRLW equation has attracted much attention of many researchers. Many mathematical and numerical studies have been developed for the MRLW equation in the literatures. Along the mathematical front, for the exact solutions via double reduction theory and Lie symmetries, the bifurcation and travelling wave solutions as well as some explicit analytic solutions obtained from dynamical systems theory, numerical solutions with high degree of accuracy by the variational iteration method and the Adomian decomposition method, we refer readers to Naz et al. (2013), Yan et al. (2012), Labidi and Omrani (2011), Khalifa et al. (2008a).

Along the numerical front, many efficient numerical methods have been developed for the MRLW equation, such as a new ten-point multisymplectic explicit numerical method (Cai 2010), Sinc-collocation method (Mokhtari and Mohammadi 2010), split least-squares mixed finite element method (Gao et al. 2015), B-spline finite element method (Gardner et al. 1997), finite difference method (Khalifa et al. 2007; Akbari and Mokhtari 2014), cubic B-spline collocation method (Khalifa et al. 2008b), quadratic B-spline collocation method (Tirmizi 2010), quadratic B-spline collocation method (Raslan 2009).

In recent works (Dehghan et al. 2009; Xie et al. 2009; Wang and Guo 2011; Wang 2014, 2015), the fourth-order compact finite difference approximation solutions to solve the Klein-Gordon equation, the Schrödinger equation and Klein-Gordon-Schrödinger equation were shown, respectively. The numerical results are encouraging. Motivated by the techniques of these works, in this paper, we propose a linearized compact conservative difference scheme with high accuracy to solve the MRLW equation (2) numerically. The presented compact difference scheme is three-level, linear-implicit and secondorder accuracy in time and fourth-order accuracy in space. By means of the matrix theory, we convert the proposed scheme into the vector difference one. The coefficient matrices of the present scheme are symmetric and tridiagonal, and Thomas algorithm can be employed to solve them effectively. Numerical example on the model problem shows that the present scheme is of high accuracy and good stability, which preserves the original conservative properties at the same time.

The rest of this paper is organized as follows. In "The high-accuracy compact conservative vector difference scheme" section, a linearized compact finite difference scheme for the MRLW equation is described. In "Discrete conservative property, estimate and solvability" section, we discuss the solvability of the scheme and the estimate of the difference solution. In "Convergence and stability of the difference scheme" section, convergence and stability of the scheme are proved by using energy method. In "Numerical experiments" section, numerical experiments are reported to test the theoretical results.

\section{The high-accuracy compact conservative vector difference scheme}

In this section, we describe a high-order linear-compact conservative difference scheme for the Eq. (2). Consider the MRLW equation

$$
u_{t}-\mu u_{x x t}+u_{x}+\alpha u^{2} u_{x}=0,
$$

with an initial condition

$$
u(x, 0)=u_{0}(x), \quad x \in\left[x_{l}, x_{r}\right]
$$


and the boundary conditions

$$
u\left(x_{l}, t\right)=u\left(x_{r}, t\right)=0, \quad t \in[0, T] .
$$

where $u_{0}(x)$ is a known smooth function.

The IBV problem (3)-(5) is known to possess the following conservative property:

$$
E(t)=\|u\|_{L_{2}}^{2}+\mu\left\|u_{x}\right\|_{L_{2}}^{2}=E(0)
$$

Let $h=\frac{x_{r}-x_{l}}{J}$ and $\tau=\frac{T}{N}$ be the uniform step size in the spatial and temporal direction, respectively. Denote $x_{j}=j h(0 \leq j \leq J), t_{n}=n \tau(0 \leq n \leq N), u_{j}^{n} \approx u\left(x_{j}, t_{n}\right)$ and $Z_{h}^{0}=\left\{u=\left(u_{j}\right) \mid u_{0}=u_{J}=0, j=0,1,2, \ldots, J\right\}$. Define

$$
\begin{aligned}
& \delta_{x} u_{j}^{n}=\frac{u_{j+1}^{n}-u_{j}^{n}}{h}, \quad \delta_{\bar{x}} u_{j}^{n}=\frac{u_{j}^{n}-u_{j-1}^{n}}{h}, \quad \delta_{\hat{x}} u_{j}^{n}=\frac{u_{j+1}^{n}-u_{j-1}^{n}}{2 h}, \quad \delta_{t} u_{j}^{n}=\frac{u_{j}^{n+1}-u_{j}^{n}}{\tau}, \\
& \delta_{\hat{t}} u_{j}^{n}=\frac{u_{j}^{n+1}-u_{j}^{n-1}}{2 \tau}, \quad \bar{u}_{j}^{n}=\frac{u_{j}^{n+1}+u_{j}^{n-1}}{2}, \\
& \mathcal{A}_{h} u_{j}^{n}=u_{j}^{n}+\frac{h^{2}}{12} \delta_{x} \delta_{\bar{x}} u_{j}^{n}=\frac{1}{12}\left(u_{j-1}^{n}+10 u_{j}^{n}+u_{j+1}^{n}\right) \\
& u_{j}^{n+\frac{1}{2}}=\frac{u_{j}^{n+1}+u_{j}^{n}}{2}, \quad \mathcal{B}_{h} u_{j}^{n}=u_{j}^{n}+\frac{h^{2}}{6} \delta_{x} \delta_{\bar{x}} u_{j}^{n}=\frac{1}{6}\left(u_{j-1}^{n}+4 u_{j}^{n}+u_{j+1}^{n}\right) .
\end{aligned}
$$

In the paper, $C$ denotes a general positive constant which may have different values in different occurrences.

For the one-order derivative $u_{x}$ and two-order derivative $u_{x x}$, we have the following formulas:

$$
u_{x}\left(x_{j}\right)=\mathcal{B}_{h}^{-1} \delta_{\hat{x}} u\left(x_{j}\right)+O\left(h^{4}\right), \quad N_{x x}\left(x_{j}\right)=\mathcal{A}_{h}^{-1} \delta_{x} \delta_{\bar{x}} u\left(x_{j}\right)+O\left(h^{4}\right), \quad(j \neq 0, J) .
$$

Omitting the high-order terms $O\left(h^{4}\right)$ in the formulas above, we consider the following three-level linear compact scheme for the IBV problem (3)-(5).

$$
\begin{aligned}
& \mathcal{A}_{h} \mathcal{B}_{h} \delta_{\hat{t}} u_{j}^{n}-\mu \mathcal{B}_{h} \delta_{x} \delta_{\bar{x}} \delta_{\hat{t}} u_{j}^{n}+\mathcal{A}_{h} \delta_{\hat{x}}\left(u_{j}^{n}\right)+\frac{1}{4} \alpha \mathcal{A}_{h}\left\{\left(\delta_{\hat{x}} \bar{u}_{j}^{n}\right)\left(u_{j}^{n}\right)^{2}+\delta_{\hat{x}}\left[\left(u_{j}^{n}\right)^{2}\left(\bar{u}_{j}^{n}\right)\right]\right\}=0 \\
& 1 \leq j \leq J-1,1 \leq n \leq N-1 \\
& \mathcal{A}_{h} \mathcal{B}_{h} \delta_{t} u_{j}^{0}-\mu \mathcal{B}_{h} \delta_{x} \delta_{\bar{x}} \delta_{t} u_{j}^{0}+\mathcal{A}_{h} \delta_{\hat{x}} u_{j}^{0+\frac{1}{2}}+\alpha \frac{1}{4} \mathcal{A}_{h}\left\{\left(\delta_{\hat{x}} u_{j}^{0+\frac{1}{2}}\right)\left(u_{j}^{0+\frac{1}{2}}\right)^{2}+\delta_{\hat{x}}\left[\left(u_{j}^{0+\frac{1}{2}}\right)^{3}\right]\right\}=0 \\
& 1 \leq j \leq J-1, \\
& u_{j}^{0}=u_{0}\left(x_{j}\right), \quad 1 \leq j \leq J \\
& u_{0}^{n}=u_{J}^{n}=0, \quad 0 \leq n \leq N .
\end{aligned}
$$

The scheme (7) is three-level and linear-implicit, so it can be easily implemented and suitable for parallel computing. 
Define

$$
\begin{aligned}
& \mathbf{u}^{n}=\left(u_{1}^{n}, u_{2}^{n}, \ldots, u_{J-1}^{n}\right)^{\mathrm{T}}, \quad \boldsymbol{M}=\left(\begin{array}{ccccc}
\frac{10}{12} & \frac{1}{12} & 0 & \cdots & 0 \\
\frac{1}{12} & \frac{10}{12} & \frac{1}{12} & \cdots & 0 \\
& \ddots & \ddots & \ddots & \\
0 & \cdots & \frac{1}{12} & \frac{10}{12} & \frac{1}{12} \\
0 & \cdots & 0 & \frac{1}{12} & \frac{10}{12}
\end{array}\right)_{(J-1) \times(J-1)} ; \\
& \boldsymbol{K}=\left(\begin{array}{ccccc}
\frac{4}{6} & \frac{1}{6} & 0 & \cdots & 0 \\
\frac{1}{6} & \frac{4}{6} & \frac{1}{6} & \cdots & 0 \\
& \ddots & \ddots & \ddots & \\
0 & \cdots & \frac{1}{6} & \frac{4}{6} & \frac{1}{6} \\
0 & \cdots & 0 & \frac{1}{6} & \frac{4}{6}
\end{array}\right)_{(J-1) \times(J-1)}
\end{aligned}
$$

Notice that $\boldsymbol{M}$ and $\boldsymbol{K}$ are two real-value symmetric positive definite matrices. Hence there exist two real-value symmetric positive definite matrices $\boldsymbol{G}$ and $\boldsymbol{H}$, such that $\boldsymbol{G}=\boldsymbol{M}^{-1}, \boldsymbol{H}=\boldsymbol{K}^{-1}$. Then (7)-(10) can be rewritten into the vector form as follows:

$$
\begin{aligned}
& \delta_{\hat{t}} \mathbf{u}^{n}-\mu \boldsymbol{G} \delta_{x} \delta_{\bar{x}} \delta_{\hat{t}} \mathbf{u}^{n}+\boldsymbol{H} \delta_{\hat{x}} \mathbf{u}^{n}+\frac{1}{4} \alpha \boldsymbol{H}\left\{\left(\delta_{\hat{x}} \overline{\mathbf{u}}^{n}\right)\left(\mathbf{u}^{n}\right)^{2}+\delta_{\hat{x}}\left[\left(\mathbf{u}^{n}\right)^{2} \overline{\mathbf{u}}^{n}\right]\right\}=0, \\
& 1 \leq n \leq N-1, \\
& \delta_{t} \mathbf{u}^{0}-\mu \boldsymbol{G} \delta_{x} \delta_{\bar{x}} \delta_{t} \mathbf{u}^{0}+\boldsymbol{H} \delta_{\hat{x}} \mathbf{u}^{0+\frac{1}{2}}+\frac{1}{4} \alpha \boldsymbol{H}\left\{\left(\delta_{\hat{x}} \mathbf{u}^{0+\frac{1}{2}}\right)\left(\mathbf{u}^{0+\frac{1}{2}}\right)^{2}\right. \\
& \left.\quad+\delta_{\hat{x}}\left[\left(\mathbf{u}^{0+\frac{1}{2}}\right)^{3}\right]\right\}=0, \\
& \mathbf{u}^{0}=\mathbf{u}_{0}, \quad 1 \leq j \leq J-1, \\
& u_{0}^{n}=u_{J}^{n}=0, \quad 0 \leq n \leq N .
\end{aligned}
$$

For convenience, the last term of (11) is defined by

$$
\kappa\left(\mathbf{u}^{n}, \overline{\mathbf{u}}^{n}\right)=\frac{1}{4} \alpha \boldsymbol{H}\left\{\left(\delta_{\hat{x}} \overline{\mathbf{u}}^{n}\right)\left(\mathbf{u}^{n}\right)^{2}+\delta_{\hat{x}}\left[\left(\mathbf{u}^{n}\right)^{2} \overline{\mathbf{u}}^{n}\right]\right\} .
$$

\section{Discrete conservative property, estimate and solvability}

In this section, we shall discuss the estimate for the difference solution and the solvability of the difference scheme (11). For $\forall v^{n}, w^{n} \in Z_{h}^{0}$, we define the discrete inner products and norms on $Z_{h}^{0}$ via:

$$
\begin{aligned}
& \left(v^{n}, w^{n}\right)=h \sum_{j=1}^{J-1} v_{j}^{n} \overline{w_{j}^{n}}, \quad\left(\delta_{x} v^{n}, \delta_{x} w^{n}\right)_{l}=h \sum_{j=0}^{J-1} \delta_{x} v_{j}^{n} \delta_{x} \overline{w_{j}^{n}}, \quad\left\|v^{n}\right\|^{2}=\left(v^{n}, v^{n}\right), \\
& \left\|\delta_{x} v^{n}\right\|=\sqrt{\left(\delta_{x} v^{n}, \delta_{x} v^{n}\right)_{l}}, \quad\left\|v^{n}\right\|_{\infty}=\max _{1 \leq j \leq J-1}\left|v_{j}^{n}\right| .
\end{aligned}
$$


To analyze the discrete conservative property and estimates of difference solution for the scheme (11)-(14), the following lemmas should be introduced.

Lemma 1 (Wang and Guo 2011) For any real value symmetric positive definite matrix $\boldsymbol{G}_{(J-1) \times(J-1)}$, then we have

$$
\begin{aligned}
& \left(\boldsymbol{G} \delta_{x} \delta_{\bar{x}} \mathbf{u}^{n}, \mathbf{u}^{n}\right)=-\left\|\boldsymbol{R} \delta_{x} \mathbf{u}^{n}\right\|^{2}, \\
& \left(\boldsymbol{G} \delta_{x} \delta_{\bar{x}}\left(\mathbf{u}^{n+1}+\mathbf{u}^{n-1}\right), \mathbf{u}^{n+1}-\mathbf{u}^{n-1}\right)=-\left(\left\|\boldsymbol{R} \delta_{x} \mathbf{u}^{n+1}\right\|^{2}-\left\|\boldsymbol{R} \delta_{x} \mathbf{u}^{n-1}\right\|^{2}\right),
\end{aligned}
$$

where $\boldsymbol{R}$ is obtained from $\mathbf{G}$ by Cholesky decomposition (Zhang 2004).

Lemma 2 (Wang 2015) On the matrices $\boldsymbol{M}$ and $\boldsymbol{K}$, there are the following results:

(1) The eigenvalues of the matrices $\boldsymbol{M}$ and $\boldsymbol{K}$ are

$$
\lambda_{M, j}=\frac{1}{12}\left(10+2 \cos \frac{j \pi}{J}\right), \quad \lambda_{K, j}=\frac{1}{6}\left(4+2 \cos \frac{j \pi}{J}\right), \quad j=1,2, \ldots, J-1 .
$$

(2) The two matrices have the same eigenvectors

$$
\mathbf{v}_{k}=\left(\sin \frac{k \pi}{J}, \sin \frac{2 k \pi}{J}, \ldots, \sin \frac{(J-1) k \pi}{J}\right)^{\mathrm{T}}, \quad k=1,2, \ldots, J-1 .
$$

Lemma 3 For real value symmetric positive definite matrices $\boldsymbol{G}_{(J-1) \times(J-1)}=\boldsymbol{M}^{-1}$ and $\boldsymbol{H}_{(J-1) \times(J-1)}=\boldsymbol{K}^{-1}$, then there exist three positive constants $C_{0} C_{1}$ and $C_{2}$ such that

$$
\begin{aligned}
& C_{0}\left\|\mathbf{u}^{n}\right\|^{2} \leq\left(\boldsymbol{G} \mathbf{u}^{n}, \mathbf{u}^{n}\right)=\left\|\boldsymbol{R} \mathbf{u}^{n}\right\|^{2} \leq C_{1}\left\|\mathbf{u}^{n}\right\|^{2}, \\
& C_{0}\left\|\mathbf{u}^{n}\right\|^{2} \leq\left(\boldsymbol{H} \mathbf{u}^{n}, \mathbf{u}^{n}\right)=\left\|\boldsymbol{S} \mathbf{u}^{n}\right\|^{2} \leq C_{2}\left\|\mathbf{u}^{n}\right\|^{2},
\end{aligned}
$$

where $C_{0}=1, C_{1}=\frac{3}{2}, C_{2}=3, \boldsymbol{R}$ and $\boldsymbol{S}$ are obtained from $\boldsymbol{G}, \boldsymbol{H}$ by Cholesky decomposition (Zhang 2004) respectively.

Proof It follows from Lemma 2 that

$$
\frac{2}{3} \leq\left|\lambda_{M, j}\right| \leq 1, \quad \frac{1}{3} \leq\left|\lambda_{K, j}\right| \leq 1
$$

This implies that

$$
1 \leq\left|\lambda_{G, j}\right| \leq \frac{3}{2}, \quad 1 \leq\left|\lambda_{H, j}\right| \leq 3
$$

Notice that $\boldsymbol{G}$ and $\boldsymbol{H}$ are also real value symmetric positive definite matrices. From Cholesky decomposition, we obtain

$$
\boldsymbol{G}=\boldsymbol{R}^{\mathrm{T}} \boldsymbol{R}, \boldsymbol{H}=\boldsymbol{S}^{\mathrm{T}} \boldsymbol{S}
$$

Then 


$$
\left(\boldsymbol{G} \mathbf{u}^{n}, \mathbf{u}^{n}\right)=\left(\boldsymbol{R} \mathbf{u}^{n}, \boldsymbol{R} \mathbf{u}^{n}\right)=\left\|\boldsymbol{R} \mathbf{u}^{n}\right\|^{2} .
$$

This together with the definition of matrix norm and (18) gives that

$$
C_{0}\left\|\mathbf{u}^{n}\right\|^{2} \leq\left\|\mathbf{R} \mathbf{u}^{n}\right\|^{2} \leq C_{1}\left\|\mathbf{u}^{n}\right\|^{2},
$$

where $C_{0}=1, C_{1}=\frac{3}{2}$. Similarly, we can also obtain

$$
C_{0}\left\|\mathbf{u}^{n}\right\|^{2} \leq\left\|S \mathbf{u}^{n}\right\|^{2} \leq C_{2}\left\|\mathbf{u}^{n}\right\|^{2}
$$

where $C_{0}=1, C_{2}=3$.

Remark 1 On the above real value symmetric positive definite matrices $\boldsymbol{G}$ and $\boldsymbol{H}$, according to Lemmas 2 and 3 , for $C$ is big enough, we can have $\left\|S \mathbf{u}^{n}\right\|^{2} \leq C\left\|\boldsymbol{R} \mathbf{u}^{n}\right\|^{2}$.

We also use the following Lemma.

Lemma 4 (Discrete Sobolev's inequality Zhou 1990) There exist two positive constants $C_{1}$ and $C_{2}$ such that

$$
\left\|u^{n}\right\|_{\infty} \leq C_{1}\left\|u^{n}\right\|+C_{2}\left\|\delta_{x} u^{n}\right\| .
$$

Theorem 1 Suppose $u_{0} \in H_{0}^{1}\left[x_{l}, x_{r}\right]$ then the scheme (11)-(14) admits the following invariant

$$
\begin{aligned}
E^{n}= & \frac{1}{2}\left(\left\|\mathbf{u}^{n+1}\right\|^{2}+\left\|\mathbf{u}^{n}\right\|^{2}\right)+\frac{1}{2} \mu\left(\left\|\boldsymbol{R} \delta_{x} \mathbf{u}^{n+1}\right\|^{2}+\left\|\boldsymbol{R} \delta_{x} \mathbf{u}^{n}\right\|^{2}\right) \\
& +h \tau \sum_{j=1}^{J-1}\left(\boldsymbol{S} \delta_{\hat{x}} u_{j}^{n}\right) \boldsymbol{S} u_{j}^{n+1} \\
= & E^{n-1}=\cdots=E^{0} .
\end{aligned}
$$

Proof Taking the inner product of (11) with $\mathbf{u}^{n+1}+\mathbf{u}^{n-1}$ (i.e. $2 \overline{\mathbf{u}}^{n}$ ) and using Lemma 1 yield

$$
\begin{aligned}
& \frac{1}{2 \tau}\left(\left\|\mathbf{u}^{n+1}\right\|^{2}-\left\|\mathbf{u}^{n-1}\right\|^{2}\right)+\frac{1}{2 \tau} \mu\left(\left\|\boldsymbol{R} \delta_{x} \mathbf{u}^{n+1}\right\|^{2}-\left\|\boldsymbol{R} \delta_{x} \mathbf{u}^{n-1}\right\|^{2}\right)+\left(\boldsymbol{H} \delta_{\hat{x}} \mathbf{u}^{n}, \mathbf{u}^{n+1}+\mathbf{u}^{n-1}\right) \\
& \quad+\left(\kappa\left(\mathbf{u}^{n}, \overline{\mathbf{u}}^{n}\right), 2 \overline{\mathbf{u}}^{n}\right)=0 .
\end{aligned}
$$

Computing the third term of the left-hand side in (24), we get

$$
\begin{aligned}
\left(\boldsymbol{H} \delta_{\hat{x}} \mathbf{u}^{n}, \mathbf{u}^{n+1}+\mathbf{u}^{n-1}\right) & =\left[\left(\boldsymbol{S} \delta_{\hat{x}} \mathbf{u}^{n}, \boldsymbol{S} \mathbf{u}^{n+1}\right)-\left(\boldsymbol{S} \mathbf{u}^{n}, \boldsymbol{S} \delta_{\hat{x}} \mathbf{u}^{n-1}\right)\right] \\
& =\left[h \sum_{j=1}^{J-1}\left(\boldsymbol{S} \delta_{\hat{x}} u_{j}^{n}\right) \boldsymbol{S} u_{j}^{n+1}-h \sum_{j=1}^{J-1}\left(\boldsymbol{S} \delta_{\hat{x}} u_{j}^{n-1}\right) \boldsymbol{S} u_{j}^{n}\right] .
\end{aligned}
$$


Computing the fourth term of the left-hand side in (24) yields

$$
\begin{aligned}
\left(\kappa\left(\mathbf{u}^{n}, \overline{\mathbf{u}}^{n}\right), 2 \overline{\mathbf{u}}^{n}\right) & =\left(\frac{1}{4} \alpha \boldsymbol{H}\left\{\left(\delta_{\hat{x}} \overline{\mathbf{u}}^{n}\right)\left(\mathbf{u}^{n}\right)^{2}+\delta_{\hat{x}}\left[\left(\mathbf{u}^{n}\right)^{2} \overline{\mathbf{u}}^{n}\right]\right\}, 2 \overline{\mathbf{u}}^{n}\right) \\
& =\frac{1}{2} \alpha\left[\left(\boldsymbol{S}\left(\delta_{\hat{x}} \overline{\mathbf{u}}^{n}\right)\left(\mathbf{u}^{n}\right)^{2}, \boldsymbol{S} \overline{\mathbf{u}}^{n}\right)-\left(\boldsymbol{S}\left(\mathbf{u}^{n}\right)^{2} \overline{\mathbf{u}}^{n}, \boldsymbol{S} \delta_{\hat{x}} \overline{\mathbf{u}}^{n}\right)\right] \\
& =\frac{1}{2} \alpha\left[\left(\boldsymbol{H}\left(\delta_{\hat{x}} \overline{\mathbf{u}}^{n}\right)\left(\mathbf{u}^{n}\right)^{2}, \overline{\mathbf{u}}^{n}\right)-\left(\boldsymbol{S}\left(\mathbf{u}^{n}\right)^{2} \overline{\mathbf{u}}^{n}, \boldsymbol{S} \delta_{\hat{x}} \overline{\mathbf{u}}^{n}\right)\right] \\
& =\frac{1}{2} \alpha\left[h \sum_{j=1}^{J-1} \boldsymbol{H}\left(\delta_{\hat{x}} \bar{u}_{j}^{n}\right)\left(u_{j}^{n}\right)^{2} \bar{u}_{j}^{n}-\left(\boldsymbol{S}\left(\mathbf{u}^{n}\right)^{2} \overline{\mathbf{u}}^{n}, \boldsymbol{S} \delta_{\hat{x}} \overline{\mathbf{u}}^{n}\right)\right] \\
& =\frac{1}{2} \alpha\left[\boldsymbol{H}\left(\delta_{\hat{x}} \overline{\mathbf{u}}^{n},\left(\mathbf{u}^{n}\right)^{2} \overline{\mathbf{u}}^{n}\right)-\left(\boldsymbol{S}\left(\mathbf{u}^{n}\right)^{2} \overline{\mathbf{u}}^{n}, \boldsymbol{S} \delta_{\hat{x}} \overline{\mathbf{u}}^{n}\right)\right] \\
& =\frac{1}{2} \alpha\left[\left(\boldsymbol{S} \delta_{\hat{x}} \overline{\mathbf{u}}^{n}, \boldsymbol{S}\left(\mathbf{u}^{n}\right)^{2} \overline{\mathbf{u}}^{n}\right)-\left(\boldsymbol{S}\left(\mathbf{u}^{n}\right)^{2} \overline{\mathbf{u}}^{n}, \boldsymbol{S} \delta_{\hat{x}} \overline{\mathbf{u}}^{n}\right)\right]=0 .
\end{aligned}
$$

It follows from (24) to (26) that

$$
\begin{aligned}
& \frac{1}{2 \tau}\left(\left\|\mathbf{u}^{n+1}\right\|^{2}-\left\|\mathbf{u}^{n-1}\right\|^{2}\right)+\frac{1}{2 \tau} \mu\left(\left\|\boldsymbol{R} \delta_{x} \mathbf{u}^{n+1}\right\|^{2}-\left\|\boldsymbol{R} \delta_{x} \mathbf{u}^{n-1}\right\|^{2}\right) \\
& \quad+h \sum_{j=1}^{J-1}\left(\boldsymbol{S} \delta_{\hat{x}} u_{j}^{n}\right) \boldsymbol{S} u_{j}^{n+1}-h \sum_{j=1}^{J-1}\left(\boldsymbol{S} \delta_{\hat{x}} u_{j}^{n-1}\right) \boldsymbol{S} u_{j}^{n}=0 .
\end{aligned}
$$

Thus

$$
E^{n}=E^{n-1}=\cdots=E^{0},
$$

where $E^{n}=\frac{1}{2}\left(\left\|\mathbf{u}^{n+1}\right\|^{2}+\left\|\mathbf{u}^{n}\right\|^{2}\right)+\frac{1}{2} \mu\left(\left\|\boldsymbol{R} \delta_{x} \mathbf{u}^{n+1}\right\|^{2}+\left\|\boldsymbol{R} \delta_{x} \mathbf{u}^{n}\right\|^{2}\right)+h \tau \sum_{j=1}^{J-1}\left(\boldsymbol{S} \delta_{\hat{x}} u_{j}^{n}\right) \boldsymbol{S} u_{j}^{n+1}$. This completes the proof of Theorem 1 .

Theorem 2 Assume that $u_{0}$ is sufficiently smooth, then there is the estimation for the solution $\mathbf{u}^{n}$ of the scheme (11)-(14): $\left\|\mathbf{u}^{n}\right\| \leq C,\left\|\delta_{x} \mathbf{u}^{n}\right\| \leq C$, which yield $\left\|\mathbf{u}^{n}\right\|_{\infty} \leq C$.

Proof It follows from (23) that

$\frac{1}{2}\left(\left\|\mathbf{u}^{n+1}\right\|^{2}+\left\|\mathbf{u}^{n}\right\|^{2}\right)+\frac{1}{2} \mu\left(\left\|\boldsymbol{R} \delta_{x} \mathbf{u}^{n+1}\right\|^{2}+\left\|\boldsymbol{R} \delta_{x} \mathbf{u}^{n}\right\|^{2}\right) \leq C+\frac{\tau}{2}\left(\left\|\boldsymbol{S} \delta_{x} \mathbf{u}^{n}\right\|^{2}+\left\|\boldsymbol{S} \mathbf{u}^{n+1}\right\|^{2}\right)$.

This together with Lemma 3 gives that

$\frac{1}{2}\left[\left(1-C_{2} \tau\right)\left\|\mathbf{u}^{n+1}\right\|^{2}+\left\|\mathbf{u}^{n}\right\|^{2}\right]+\frac{1}{2}\left[\mu\left\|\boldsymbol{R} \delta_{x} \mathbf{u}^{n+1}\right\|^{2}+\left(C_{0} \mu-C_{2} \tau\right)\left\|\delta_{x} \mathbf{u}^{n}\right\|^{2}\right] \leq C$.

Let $\tau$ be small enough, such that $1-C_{2} \tau>0, C_{0} \mu-C_{2} \beta \tau>0$, then we obtain from (30) that

$$
\left\|\mathbf{u}^{n}\right\| \leq C, \quad\left\|\delta_{x} \mathbf{u}^{n}\right\| \leq C
$$


An application of Lemma 4 yields

$$
\left\|\mathbf{u}^{n}\right\|_{\infty} \leq C
$$

Remark 2 Theorem 2 implies that scheme (11)-(14) is unconditionally stable.

Theorem 3 The difference scheme (11) is uniquely solvable.

Proof By the mathematical induction. It is obvious that $\mathbf{u}^{0}$ is uniquely determined by (13). We can choose a fourth-order method to compute $\mathbf{u}^{1}$ [such as C-N scheme (12)]. Assuming that $\mathbf{u}^{1}, \ldots, \mathbf{u}^{n}$ are uniquely solvable, consider $\mathbf{u}^{n+1}$ in (11) which satisfies

$$
\frac{1}{2 \tau} \mathbf{u}^{n+1}-\frac{1}{2 \tau} \mu \boldsymbol{G} \delta_{x} \delta_{\bar{x}} \mathbf{u}^{n+1}+\frac{1}{8} \alpha \boldsymbol{H}\left[\delta_{\hat{x}} \mathbf{u}^{n+1}\left(\mathbf{u}^{n}\right)^{2}+\delta_{\hat{x}}\left(\left(\mathbf{u}^{n}\right)^{2} \mathbf{u}^{n+1}\right)\right]=0 .
$$

Doing in (33) the inner product of with $\mathbf{u}^{n+1}$ and using Lemma 1 yield

$$
\frac{1}{2 \tau}\left\|\mathbf{u}^{n+1}\right\|^{2}+\frac{1}{2 \tau} \mu\left\|\boldsymbol{R} \delta_{x} \mathbf{u}^{n+1}\right\|^{2}+\left(\phi\left(\mathbf{u}^{n}, \mathbf{u}^{n+1}\right), \mathbf{u}^{n+1}\right)=0,
$$

where $\phi\left(\mathbf{u}^{n}, \mathbf{u}^{n+1}\right)=\frac{1}{8} \alpha \boldsymbol{H}\left[\delta_{\hat{x}} \mathbf{u}^{n+1}\left(\mathbf{u}^{n}\right)^{2}+\delta_{\hat{x}}\left(\left(\mathbf{u}^{n}\right)^{2} \mathbf{u}^{n+1}\right)\right]$.

Similarly to the proof of (26), we obtain

$$
\left(\phi\left(\mathbf{u}^{n}, \mathbf{u}^{n+1}\right), \mathbf{u}^{n+1}\right)=0
$$

This together with (34) gives that

$$
\frac{1}{2 \tau}\left\|\mathbf{u}^{n+1}\right\|^{2}+\frac{1}{2 \tau} \mu\left\|\boldsymbol{R} \delta_{x} \mathbf{u}^{n+1}\right\|^{2}=0
$$

This implies that there uniquely exists trivial solution satisfying Eq. (33). Hence, $\mathbf{u}^{n+1}$ in (11) is uniquely solvable. This completes the proof of Theorem 3.

\section{Convergence and stability of the difference scheme}

First, we shall consider the truncation error of the difference scheme (11)-(14). Let $v_{j}^{n}=u\left(x_{j}, t_{n}\right)$. We define the truncation error as follows:

$$
\begin{aligned}
\boldsymbol{r}^{n}= & \delta_{\hat{t}} \mathbf{v}^{n}-\mu \boldsymbol{G} \delta_{x} \delta_{\hat{x}} \delta_{\hat{t}} \mathbf{v}^{n}+\boldsymbol{H} \delta_{\hat{x}} \mathbf{v}^{n}+\frac{1}{4} \alpha \boldsymbol{H}\left\{\left(\delta_{\hat{x}} \overline{\mathbf{v}}^{n}\right)\left(\mathbf{v}^{n}\right)^{2}+\delta_{\hat{x}}\left[\left(\mathbf{v}^{n}\right)^{2} \overline{\mathbf{v}}^{n}\right]\right\} \\
& 1 \leq n \leq N-1, \\
\boldsymbol{\sigma}^{0}= & \delta_{t} \mathbf{v}^{0}-\mu \boldsymbol{G} \delta_{x} \delta_{\bar{x}} \delta_{t} \mathbf{v}^{0}+\boldsymbol{H} \delta_{\hat{x}} \mathbf{v}^{0+\frac{1}{2}}+\frac{1}{4} \alpha \boldsymbol{H}\left\{\left(\delta_{\hat{x}} \mathbf{v}^{0+\frac{1}{2}}\right)\left(\mathbf{v}^{0+\frac{1}{2}}\right)^{2}\right. \\
& \left.+\delta_{\hat{x}}\left[\left(\mathbf{v}^{0+\frac{1}{2}}\right)^{3}\right]\right\}, \\
& \mathbf{v}^{0}=\mathbf{u}_{0}, \quad 1 \leq j \leq J-1, \\
v_{0}^{n}= & v_{J}^{n}=0, \quad 0 \leq n \leq N .
\end{aligned}
$$


Using Taylor expansion and considering the construction of the difference scheme of (7)-(10), we know the accuracy of (7)-(10) is $O\left(\tau^{2}+h^{4}\right)$. The scheme (11)-(14) is equivalent to (7)-(10). Then we have that $\left|\boldsymbol{r}^{n}\right|=O\left(\tau^{2}+h^{4}\right),\left|\sigma^{0}\right|=O\left(\tau^{2}+h^{4}\right)$ hold if $\tau, h \rightarrow 0$.

Next, we shall discuss the convergence and stability of the scheme (11)-(14).

Lemma 5 (Discrete Gronwall inequality Zhou 1990) Suppose that the discrete mesh function $\left\{w^{n} \mid n=1,2, \ldots, N ; N \tau=T\right\}$ satisfies recurrence formula

$$
w^{n}-w^{n-1} \leq A \tau w^{n}+B \tau w^{n-1}+C_{n} \tau,
$$

where $A, B$ and $C_{n}(n=1, \ldots, N)$ are nonnegative constants. Then

$$
\left\|w^{n}\right\|_{\infty} \leq\left(w^{0}+\tau \sum_{k=1}^{N} C_{k}\right) e^{2(A+B) T},
$$

where $\tau$ is small, such that $(A+B) \tau \leq \frac{N-1}{2 N}(N>1)$.

Theorem 4 Assume that $u_{0}$ is sufficiently smooth and $u(x, t) \in C_{x, t}^{5,3}$, then the solution $\mathbf{u}^{n}$ of the scheme (10)-(12) converges to the solution of the IBV problem (3)-(5) and the rate of convergence is $O\left(\tau^{2}+h^{4}\right)$ by the $\|\cdot\|_{\infty}$ norm.

Proof Let $\mathbf{e}^{n}=\mathbf{v}^{n}-\mathbf{u}^{n}$. From (37) to (40) and (11) to (14), we have

$$
\begin{aligned}
\boldsymbol{r}^{n}= & \delta_{\hat{t}} \mathbf{e}^{n}-\mu \boldsymbol{G} \delta_{x} \delta_{\bar{x}} \delta_{\hat{t}} \mathbf{e}^{n}+\boldsymbol{H} \delta_{\hat{x}} \mathbf{e}^{n}+\frac{1}{4} \alpha \boldsymbol{H}\left\{\left(\delta_{\hat{x}} \overline{\mathbf{v}}^{n}\right)\left(\mathbf{v}^{n}\right)^{2}+\delta_{\hat{x}}\left[\left(\mathbf{v}^{n}\right)^{2} \overline{\mathbf{v}}^{n}\right]\right\} \\
& -\frac{1}{4} \alpha \boldsymbol{H}\left\{\left(\delta_{\hat{x}} \overline{\mathbf{u}}^{n}\right)\left(\mathbf{u}^{n}\right)^{2}+\delta_{\hat{x}}\left[\left(\mathbf{u}^{n}\right)^{2} \overline{\mathbf{u}}^{n}\right]\right\}, \quad 1 \leq n \leq N-1 \\
\boldsymbol{\sigma}^{0}= & \delta_{t} \mathbf{e}^{0}-\mu \boldsymbol{G} \delta_{x} \delta_{\bar{x}} \delta_{t} \mathbf{e}^{0}+\boldsymbol{H} \delta_{\hat{x}} \mathbf{e}^{0+\frac{1}{2}}+\frac{1}{4} \alpha \boldsymbol{H}\left\{\left(\delta_{\hat{x}} \mathbf{v}^{0+\frac{1}{2}}\right)\left(\mathbf{v}^{0+\frac{1}{2}}\right)^{2}+\delta_{\hat{x}}\left[\left(\mathbf{v}^{0+\frac{1}{2}}\right)^{3}\right]\right\} \\
& -\frac{1}{4} \alpha \boldsymbol{H}\left\{\left(\delta_{\hat{x}} \mathbf{u}^{0+\frac{1}{2}}\right)\left(\mathbf{u}^{0+\frac{1}{2}}\right)^{2}+\delta_{\hat{x}}\left[\left(\mathbf{u}^{0+\frac{1}{2}}\right)^{3}\right]\right\} \\
\mathbf{e}^{0}= & 0, \quad 1 \leq j \leq J-1 \\
e_{0}^{n}= & e_{J}^{n}=0, \quad 0 \leq n \leq N
\end{aligned}
$$

Doing in (41) the inner product with $2 \overline{\mathbf{e}}^{n}$ (i.e. $\mathbf{e}^{n+1}+\mathbf{e}^{n-1}$ ) and using Lemma 1, we obtain

$$
\begin{aligned}
\left(\boldsymbol{r}^{n}, 2 \overline{\mathbf{e}}^{n}\right)= & \frac{1}{2 \tau}\left(\left\|\mathbf{e}^{n+1}\right\|^{2}-\left\|\mathbf{e}^{n-1}\right\|^{2}\right)+\frac{1}{2 \tau} \mu\left(\left\|\boldsymbol{R} \delta_{x} \mathbf{e}^{n+1}\right\|^{2}-\left\|\boldsymbol{R} \delta_{x} \mathbf{e}^{n-1}\right\|^{2}\right) \\
& +h \sum_{j=1}^{J-1}\left(\boldsymbol{S} \delta_{\hat{x}} e_{j}^{n}\right) \boldsymbol{S}\left(e_{j}^{n+1}+e_{j}^{n-1}\right)+\left(P+Q, 2 \overline{\mathbf{e}}^{n}\right),
\end{aligned}
$$

where $P=\frac{1}{4} \alpha \boldsymbol{H}\left[\left(\delta_{\hat{x}} \overline{\mathbf{v}}^{n}\right)\left(\mathbf{v}^{n}\right)^{2}-\left(\delta_{\hat{x}} \overline{\mathbf{u}}^{n}\right)\left(\mathbf{u}^{n}\right)^{2}\right], Q=\frac{1}{4} \alpha \boldsymbol{H}\left\{\left[\delta_{\hat{x}}\left[\left(\mathbf{v}^{n}\right)^{2} \overline{\mathbf{v}}^{n}\right]-\delta_{\hat{x}}\left[\left(\mathbf{u}^{n}\right)^{2} \overline{\mathbf{u}}^{n}\right]\right\}\right.$. 
Computing the fourth term of right-hand side of (45) and using Theorem 2, Lemma 3 yield

$$
\begin{aligned}
\left(P, 2 \overline{\mathbf{e}}^{n}\right) & =\frac{1}{2} \alpha\left(\boldsymbol{H}\left[\left(\delta_{\hat{x}} \overline{\mathbf{v}}^{n}\right)\left(\mathbf{v}^{n}\right)^{2}-\left(\delta_{\hat{x}} \overline{\mathbf{u}}^{n}\right)\left(\mathbf{u}^{n}\right)^{2}\right], \overline{\mathbf{e}}^{n}\right) \\
& =\frac{1}{2} \alpha\left(\boldsymbol{H}\left[\left(\delta_{\hat{x}} \overline{\mathbf{e}}^{n}\right)\left(\mathbf{v}^{n}\right)^{2}+\left(\delta_{\hat{x}} \overline{\mathbf{u}}^{n}\right)\left(\left(\mathbf{v}^{n}\right)^{2}-\left(\mathbf{u}^{n}\right)^{2}\right)\right], \overline{\mathbf{e}}^{n}\right) \\
& =\frac{1}{2} \alpha h\left\{\sum_{j=1}^{J-1} \boldsymbol{S}\left(\delta_{\hat{x}} \bar{e}_{j}^{n}\right)\left(v_{j}^{n}\right)^{2} \boldsymbol{S} \bar{e}_{j}^{n}+\sum_{j=1}^{J-1} \boldsymbol{S}\left(\delta_{\hat{x}} \bar{u}_{j}^{n}\right)\left[\left(v_{j}^{n}\right)^{2}-\left(u_{j}^{n}\right)^{2}\right] \boldsymbol{S} \bar{e}_{j}^{n}\right\} \\
& =\frac{1}{2} \alpha h \sum_{j=1}^{J-1} \boldsymbol{S}\left(\delta_{\hat{x}} \bar{e}_{j}^{n}\right)\left(v_{j}^{n}\right)^{2} \boldsymbol{S} \bar{e}_{j}^{n}+\frac{1}{2} \alpha h \sum_{j=1}^{J-1} \boldsymbol{S}\left(\delta_{\hat{x}} \bar{u}_{j}^{n}\right)\left[e_{j}^{n}\left(v_{j}^{n}-u_{j}^{n}\right)\right] \boldsymbol{S} \bar{e}_{j}^{n} \\
& \leq C\left(\left\|\boldsymbol{S} \delta_{\hat{x}} \overline{\mathbf{e}}^{n}\right\|^{2}+\left\|\boldsymbol{S} \mathbf{e}^{n}\right\|^{2}+\left\|\boldsymbol{S} \overline{\mathbf{e}}^{n}\right\|^{2}\right) \\
& \leq C\left(\left\|\boldsymbol{R} \delta_{x} \mathbf{e}^{n+1}\right\|^{2}+\left\|\boldsymbol{R} \delta_{x} \mathbf{e}^{n-1}\right\|^{2}+\left\|\mathbf{e}^{n+1}\right\|^{2}+\left\|\mathbf{e}^{n}\right\|^{2}+\left\|\mathbf{e}^{n-1}\right\|^{2}\right) .
\end{aligned}
$$

Similarly, we have that

$$
\left(Q, 2 \bar{e}^{n}\right) \leq C\left(\left\|\boldsymbol{R} \delta_{x} \mathbf{e}^{n+1}\right\|^{2}+\left\|\boldsymbol{R} \delta_{x} \mathbf{e}^{n-1}\right\|^{2}+\left\|\mathbf{e}^{n+1}\right\|^{2}+\left\|\mathbf{e}^{n}\right\|^{2}+\left\|\mathbf{e}^{n-1}\right\|^{2}\right) .
$$

In addition, it is obvious that

$$
\begin{aligned}
\left(\boldsymbol{r}^{n}, 2 \overline{\mathbf{e}}^{n}\right) \leq\left\|\boldsymbol{r}^{n}\right\|^{2}+\frac{1}{2}\left(\left\|\mathbf{e}^{n+1}\right\|^{2}+\left\|\mathbf{e}^{n-1}\right\|^{2}\right) & \\
h \sum_{j=1}^{J-1}\left(\boldsymbol{S} \delta_{\hat{x}} e_{j}^{n}\right) \boldsymbol{S}\left(e_{j}^{n+1}+e_{j}^{n-1}\right) & \leq\left\|\boldsymbol{S} \delta_{x} \mathbf{e}^{n}\right\|^{2}+\frac{1}{2}\left(\left\|\mathbf{e}^{n+1}\right\|^{2}+\left\|\mathbf{e}^{n-1}\right\|^{2}\right) \\
& \leq C\left\|\boldsymbol{R} \delta_{x} \mathbf{e}^{n}\right\|^{2}+\frac{1}{2}\left(\left\|\mathbf{e}^{n+1}\right\|^{2}+\left\|\mathbf{e}^{n-1}\right\|^{2}\right) .
\end{aligned}
$$

It follows from (45) to (49) that

$$
\begin{aligned}
& \frac{1}{2 \tau}\left(\left\|\mathbf{e}^{n+1}\right\|^{2}-\left\|\mathbf{e}^{n-1}\right\|^{2}\right)+\frac{1}{2 \tau}\left(\left\|\boldsymbol{R} \delta_{x} \mathbf{e}^{n+1}\right\|^{2}-\left\|\boldsymbol{R} \delta_{x} \mathbf{e}^{n-1}\right\|^{2}\right) \\
& \quad \leq\left\|\boldsymbol{r}^{n}\right\|^{2}+C\left[\left\|\boldsymbol{R} \delta_{x} \mathbf{e}^{n+1}\right\|^{2}+\left\|\boldsymbol{R} \delta_{x} \mathbf{e}^{n}\right\|^{2}+\left\|\boldsymbol{R} \delta_{x} \mathbf{e}^{n-1}\right\|^{2}+\left\|\mathbf{e}^{n+1}\right\|^{2}+\left\|\mathbf{e}^{n}\right\|^{2}+\left\|\mathbf{e}^{n-1}\right\|^{2}\right] .
\end{aligned}
$$

Let $B^{n}=\frac{1}{2}\left(\left\|\mathbf{e}^{n+1}\right\|^{2}+\left\|\mathbf{e}^{n}\right\|^{2}\right)+\frac{1}{2}\left(\left\|\boldsymbol{R} \delta_{x} \mathbf{e}^{n+1}\right\|^{2}+\left\|\boldsymbol{R} \delta_{x} \mathbf{e}^{n}\right\|^{2}\right)$, then (50) can be written as follows:

$$
B^{n}-B^{n-1} \leq \tau\left\|\boldsymbol{r}^{n}\right\|^{2}+C \tau\left(B^{n}+B^{n-1}\right) .
$$

By Lemma 5, it can immediately be obtained that

$$
B^{n} \leq\left(B^{0}+T \sup _{l \leq n \leq N}\left\|\boldsymbol{r}^{n}\right\|^{2}\right) e^{C T} .
$$


Taking the inner product in (42) with $\mathbf{e}^{1}$, we have

$$
\left(\boldsymbol{\sigma}^{0}, \mathbf{e}^{1}\right)=\frac{1}{\tau}\left\|\mathbf{e}^{1}\right\|^{2}+\frac{1}{\tau} \mu\left\|\boldsymbol{R} \delta_{x} \mathbf{e}^{1}\right\|^{2}+\frac{1}{2}\left(\boldsymbol{H} \delta_{\hat{x}} \mathbf{e}^{1}, \mathbf{e}^{1}\right)+\left(I, \mathbf{e}^{1}\right)+\left(I I, \mathbf{e}^{1}\right),
$$

where $I=\frac{1}{4} \alpha \boldsymbol{H}\left[\left(\delta_{\hat{x}} \mathbf{v}^{0+\frac{1}{2}}\right)\left(\mathbf{v}^{0+\frac{1}{2}}\right)^{2}-\left(\delta_{\hat{x}} \mathbf{u}^{0+\frac{1}{2}}\right)\left(\mathbf{u}^{0+\frac{1}{2}}\right)^{2}\right] I I=\frac{1}{4} \alpha \boldsymbol{H}\left\{\delta_{\hat{x}}\left[\left(\mathbf{v}^{0+\frac{1}{2}}\right)^{3}\right]-\delta_{\hat{x}}\left[\left(\mathbf{u}^{0+\frac{1}{2}}\right)^{3}\right]\right\}$.

Notice that

$$
\frac{1}{2}\left(\boldsymbol{H} \delta_{\hat{x}} \mathbf{e}^{1}, \mathbf{e}^{1}\right)=0
$$

From the discrete initial condition (43), we know that $\mathbf{e}^{0}=0$. Computing the fourth term of right-hand side in (53) and using Theorem 2, Lemma 3 yield

$$
\begin{aligned}
\left(I, \mathbf{e}^{1}\right) & =\left(\frac{1}{4} \alpha \boldsymbol{H}\left[\left(\delta_{\hat{x}} \mathbf{v}^{0+\frac{1}{2}}\right)\left(\mathbf{v}^{0+\frac{1}{2}}\right)^{2}-\left(\delta_{\hat{x}} \mathbf{u}^{0+\frac{1}{2}}\right)\left(\mathbf{u}^{0+\frac{1}{2}}\right)^{2}\right], \mathbf{e}^{1}\right) \\
& =\frac{1}{8} \alpha\left(\boldsymbol{S}\left[\left(\delta_{\hat{x}} \mathbf{e}^{1}\right)\left(\mathbf{v}^{0+\frac{1}{2}}\right)^{2}+\left(\delta_{\hat{x}} \mathbf{u}^{0+\frac{1}{2}}\right) \mathbf{e}^{1}\left(\mathbf{v}^{0+\frac{1}{2}}+\mathbf{u}^{0+\frac{1}{2}}\right)\right], \boldsymbol{S} \mathbf{e}^{1}\right) \\
& \leq C\left(\left\|\boldsymbol{S} \delta_{x} \mathbf{e}^{1}\right\|^{2}+\left\|\boldsymbol{S} \mathbf{e}^{1}\right\|^{2}\right) \\
& \leq C\left(\left\|\delta_{x} \mathbf{e}^{1}\right\|^{2}+\left\|\mathbf{e}^{1}\right\|\right) .
\end{aligned}
$$

Similarly, we get

$$
\left(I I, \mathbf{e}^{1}\right) \leq C\left(\left\|\delta_{x} \mathbf{e}^{1}\right\|^{2}+\left\|\mathbf{e}^{1}\right\|\right)
$$

It follows from (53) to (56) that

$$
\frac{1}{\tau}\left\|\mathbf{e}^{1}\right\|^{2}+\frac{1}{\tau} \mu\left\|\boldsymbol{R} \delta_{x} \mathbf{e}^{1}\right\|^{2} \leq\left(\boldsymbol{\sigma}^{0}, \mathbf{e}^{1}\right)+C\left(\left\|\delta_{x} \mathbf{e}^{1}\right\|^{2}+\left\|\mathbf{e}^{1}\right\|\right) .
$$

This together with $\left(\sigma^{0}, \mathbf{e}^{1}\right) \leq \frac{1}{2}\left(\left\|\sigma^{0}\right\|^{2}+\left\|\mathbf{e}^{1}\right\|^{2}\right)$ and Lemma 3 gives that

$$
\left(\frac{1}{\tau}-\frac{1}{2}-C\right)\left\|\mathbf{e}^{1}\right\|^{2}+\left(\frac{1}{\tau} C_{0} \mu-C\right)\left\|\delta_{x} \mathbf{e}^{1}\right\|^{2} \leq \frac{1}{2}\left\|\sigma^{0}\right\|^{2} .
$$

Let $\tau$ be small enough, such that $\frac{1}{\tau}-\frac{1}{2}-C>0, \frac{1}{\tau} C_{0} \mu-C>0$. Then we obtain from (58) that

$$
\left\|\mathbf{e}^{1}\right\|^{2} \leq K_{1}\left[O\left(\tau^{2}+h^{4}\right)\right]^{2}, \quad\left\|\delta_{x} \mathbf{e}^{1}\right\|^{2} \leq K_{2}\left[O\left(\tau^{2}+h^{4}\right)\right]^{2}
$$

where $K_{1}=\frac{1}{\frac{2}{\tau}-1-2 C}, K_{2}=\frac{1}{\frac{2}{\tau} C_{0} \mu-2 C}$.

It follows from Lemma 3 that

$$
\left\|\boldsymbol{R} \delta_{x} \mathbf{e}^{1}\right\|^{2} \leq C_{1} K_{2}\left[O\left(\tau^{2}+h^{4}\right)\right]^{2}
$$


This implies that

$$
B^{0}=\left[O\left(\tau^{2}+h^{4}\right)\right]^{2} .
$$

It follows from (52) that

$$
B^{n} \leq C\left[O\left(\tau^{2}+h^{4}\right)\right]^{2},
$$

which together with Lemmas 3 and 4 , and the definition of $B^{n}$ gives that

$$
\left\|\mathbf{e}^{n}\right\|_{\infty} \leq C \cdot O\left(\tau^{2}+h^{4}\right)
$$

This completes the proof of Theorem 4.

Similarly, we can prove stability of the difference solution.

Theorem 5 Under the conditions of Theorem 4, the solution $\mathbf{u}^{n}$ of the scheme (11)-(14) is unconditionally stable by the $\|\cdot\|_{\infty}$ norm.

\section{Numerical experiments}

In this section, we conduct some numerical experiments to verify our theoretical results obtained in the previous sections. In order to test whether the present scheme (11)-(14) exhibits the expect convergence rates in time and in space, we will measure the accuracy of the proposed scheme using the square norm errors and the maximum norm errors defined by

$$
e_{\epsilon_{1}}^{n}=\left\|\mathbf{v}^{n}-\mathbf{u}^{n}\right\|_{\infty}, \quad e_{\epsilon_{2}}^{n}=\left\|\mathbf{v}^{n}-\mathbf{u}^{n}\right\| .
$$

The exact solution of the IBV problem (3)-(5) has the following form (Gardner et al. 1997):

$$
u(x, t)=\sqrt{\frac{6 c}{\alpha}} \operatorname{sech}\left[\sqrt{\frac{c}{\mu(c+1)}}\left(x-(c+1) t-x_{0}\right)\right],
$$

where $x_{0}, c$ are arbitrary constants.

It follows from (64) that the initial-boundary value problem (3)-(5) is consistent to the initial value problem (3) and (4) for $-x_{l} \gg 0, x_{r} \gg 0$. In the following numerical experiments, we take $x_{l}=-40, x_{r}=60, T=10$.

The IBV problem (3)-(5) has another two invariants (Gardner et al. 1997):

$$
Q(t)=\int_{x_{l}}^{x_{r}} u(x, t) \mathrm{d} x \simeq h \sum_{j=1}^{J-1} u_{j}^{n},
$$




$$
\tilde{E}(t)=\int_{x_{l}}^{x_{r}}\left[u^{4}-\frac{6 \mu}{\alpha} u_{x}^{2}\right] \mathrm{d} x \simeq h \sum_{j=1}^{J-1}\left[\left(u_{j}^{n}\right)^{4}-\frac{6 \mu}{\alpha}\left(\left(u_{x}\right)_{j}^{n}\right)^{2}\right] .
$$

The initial condition of the studied model is obtained from (64) with the parameters $x_{0}, c, \alpha$ and $\mu$ :

$$
u(x, 0)=\sqrt{\frac{6 c}{\alpha}} \operatorname{sech}\left[\sqrt{\frac{c}{\mu(c+1)}}\left(x-x_{0}\right)\right] .
$$

In computations, we always choose the parameter $x_{0}=0$. Take the parameters $c=\alpha=\mu=1$. To verify the accuracy $O\left(\tau^{2}+h^{4}\right)$ in the spatial direction, we take $\tau=h^{2}$. And we choose $h$ small enough to verify the second-order accuracy in the temporal direction. The convergence order figure of $\log \left(e^{n}\right)-\log (h)$ with $\tau=h^{2}$ and the one of $\log \left(e^{n}\right)-\log (\tau)$ with $h$ small enough are given in Figs. 1 and 2 under various mesh steps $h$ and $\tau$ at $t=10$. From Figs. 1 and 2, it is obvious that the scheme (11)-(14) is convergent in maximum norm, and the convergence order is $O\left(\tau^{2}+h^{4}\right)$.

The errors in the sense of $L_{\infty}$-norm and $L_{2}$-norm of the numerical solutions $\mathbf{u}^{n}$ of the scheme (11) are listed on Tables 1 and 2. Tables 1 and 2 shows good stability of the numerical solutions and also verify the scheme in present paper is efficient and of high accuracy.

We have shown in Theorem 1 that the numerical solution $\mathbf{u}^{n}$ of the scheme (11) satisfies the conservative property (23). The values of $E^{n}, Q \tilde{E}$ for the scheme (11) are presented in Table 3 under steps $h=0.1$ and $\tau=0.01$. It is easy to see from Table 3 that the scheme (11) preserves the discrete conservative properties very well, thus it can be used to computing for a long time.

The curves of the solitary wave with time computed by scheme (11) with $h=0.05$ and $\tau=0.0025$ are given in Fig. 3; the waves at $t=5$ and 10 agree with the ones at $t=0$ quite well, which also demonstrate the accuracy and efficiency of the scheme in present paper.

To compare the numerical results with other results shown in previous studies, we denote the proposed scheme in Akbari and Mokhtari (2014) as Scheme I with $p=2, \mu=\varepsilon=1$ and $d=\frac{1}{3}$. Denote the present scheme (11) with $c=\frac{1}{3}, \alpha=\mu=1$ as Scheme II. The corresponding errors in the sense of $L_{\infty}$-norm and CPU time are listed on Table 4 under different mesh steps $h$ and $\tau$. From Table 4 , we get that a fourth-order three-level linear scheme as accurate as Scheme I which is a two-level one.

\section{Conclusion}

In this paper, an attempt has been made to construct a new numerical scheme to solve the initial-boundary problem of the MRLW equation, which has the following 


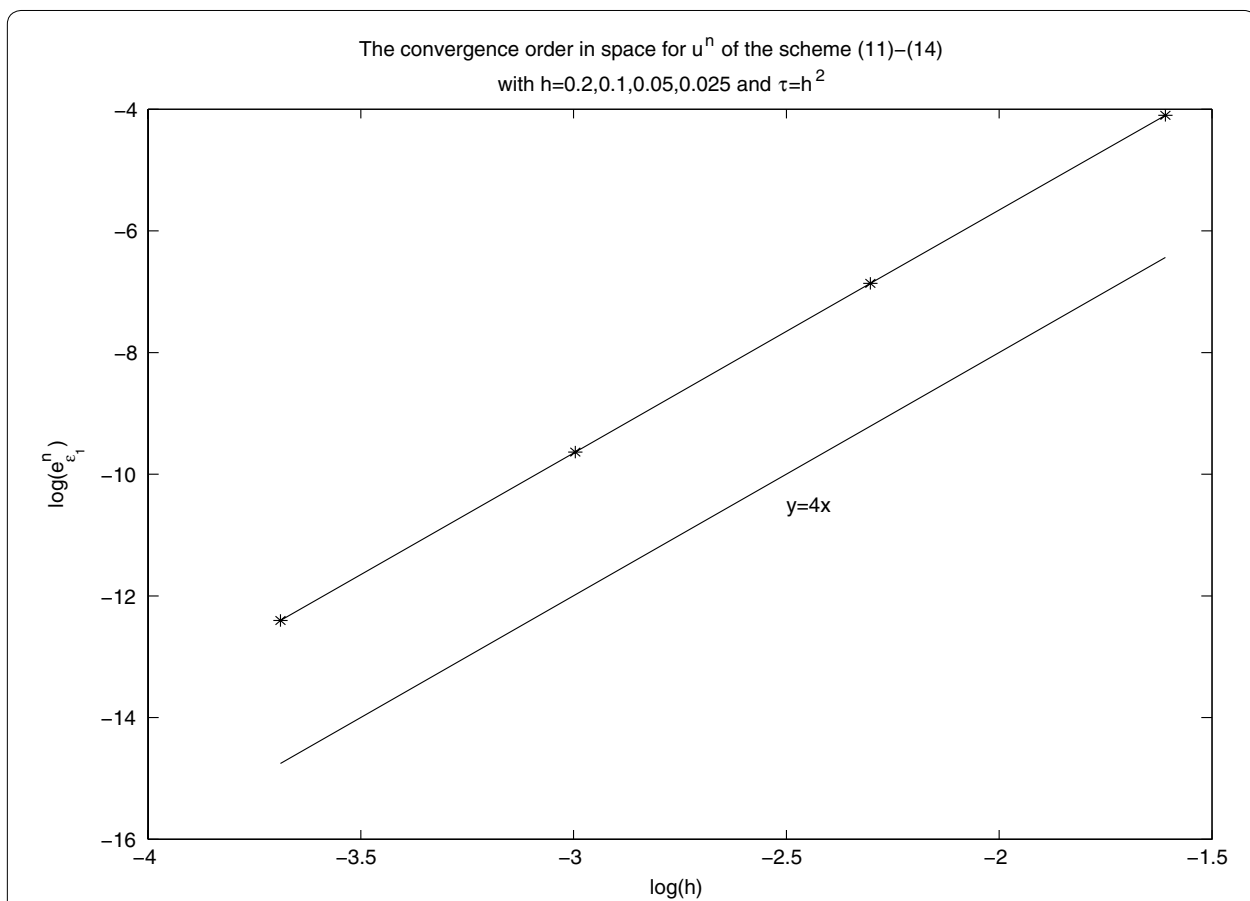

Fig. 1 Spatial convergence order in maximal norm for $u^{n}$ at $t=10$ with different $h$ and $\tau$ computed by the scheme (11)-(14)

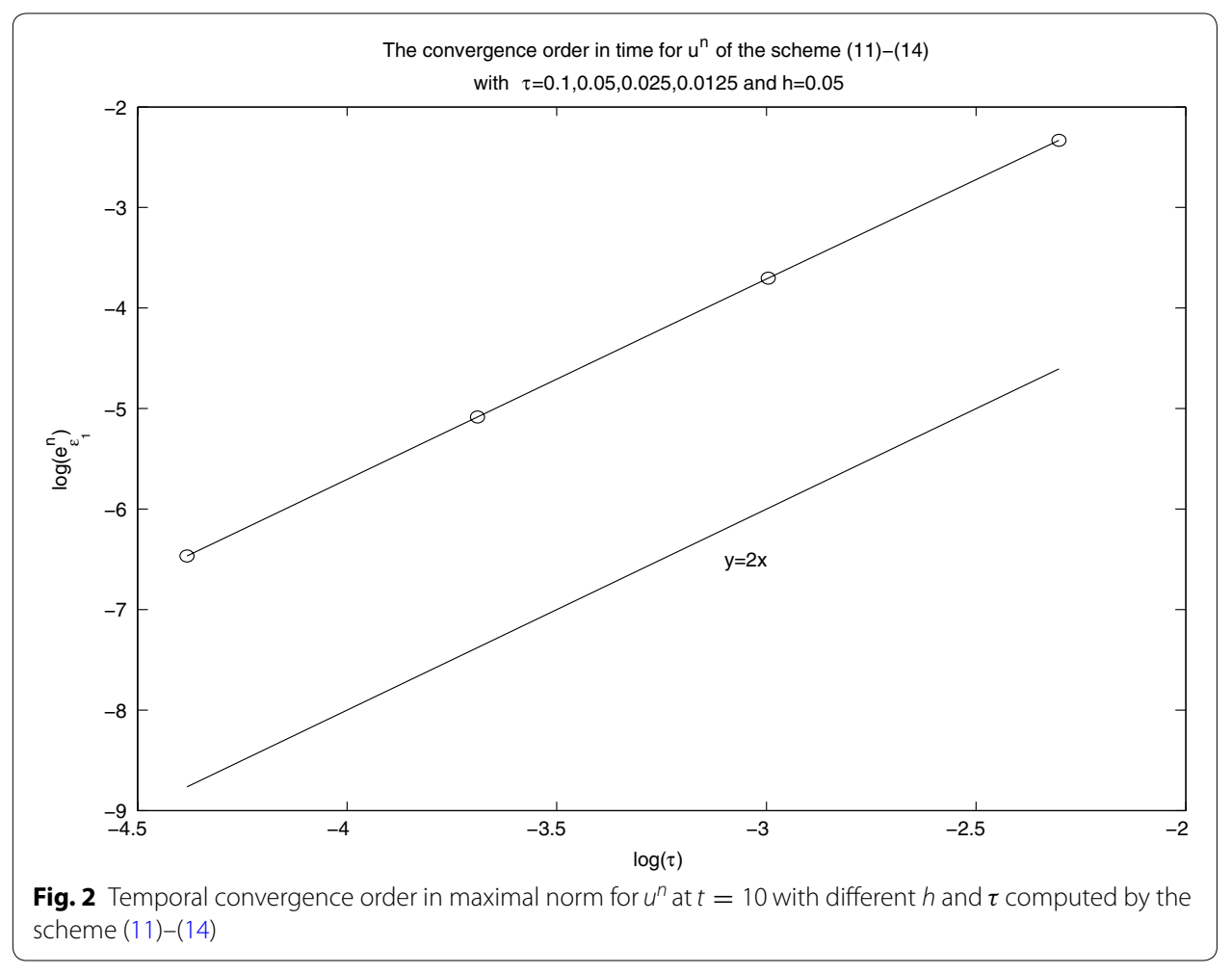


Table 1 The errors for numerical solutions $u^{n}$ of the scheme (11)-(14) at different time $t$ with various $h$ and $\tau=h^{2}$

\begin{tabular}{|c|c|c|c|c|c|c|c|c|}
\hline \multirow[t]{2}{*}{$t$} & \multicolumn{2}{|l|}{$h=0.2$} & \multicolumn{2}{|l|}{0.1} & \multicolumn{2}{|l|}{0.05} & \multicolumn{2}{|l|}{0.025} \\
\hline & $e_{\epsilon_{1}}^{n}$ & $e_{\epsilon_{2}}^{n}$ & $e_{\epsilon_{1}}^{n}$ & $e_{\epsilon_{2}}^{n}$ & $e_{\epsilon_{1}}^{n}$ & $e_{\epsilon_{2}}^{n}$ & $e_{\epsilon_{1}}^{n}$ & $e_{\epsilon_{2}}^{n}$ \\
\hline 2 & $3.7504 \mathrm{e}-3$ & $6.8705 e-3$ & $2.3827 e-4$ & $4.3361 \mathrm{e}-4$ & $1.4930 \mathrm{e}-5$ & $2.7153 e-5$ & $9.3409 \mathrm{e}-7$ & $1.6997 \mathrm{e}-6$ \\
\hline 4 & $7.1325 e-3$ & $1.3403 e-2$ & $4.5011 \mathrm{e}-4$ & $8.438 \mathrm{e}-4$ & $2.8167 e-5$ & $5.2812 \mathrm{e}-5$ & $1.7639 \mathrm{e}-6$ & $3.3089 e-6$ \\
\hline 6 & $1.0303 e-2$ & $1.9666 \mathrm{e}-2$ & $6.5000 \mathrm{e}-4$ & $1.2367 e-3$ & $4.0665 e-5$ & $7.7372 \mathrm{e}-5$ & $2.5487 e-6$ & $4.8528 \mathrm{e}-6$ \\
\hline 0 & $1.3432 \mathrm{e}-2$ & $2.5820 e-2$ & $8.4767 e-4$ & $1.6227 \mathrm{e}-3$ & $5.3023 e-5$ & $1.0151 \mathrm{e}-4$ & $3.3268 \mathrm{e}-6$ & $6.3733 e-6$ \\
\hline 10 & $1.6551 \mathrm{e}-2$ & $3.1935 e-2$ & $1.0447 \mathrm{e}-3$ & $2.0063 e-3$ & $6.5345 e-5$ & $1.2549 \mathrm{e}-4$ & $4.1045 e-6$ & $7.8878 e-6$ \\
\hline
\end{tabular}

Table 2 The errors for numerical solutions $u^{n}$ of the scheme (11)-(14) at different time $t$ with various $\tau$ when $h=0.05$

\begin{tabular}{|c|c|c|c|c|c|c|c|c|}
\hline \multirow[t]{2}{*}{$t$} & \multicolumn{2}{|l|}{$\tau=0.1$} & \multicolumn{2}{|l|}{0.05} & \multicolumn{2}{|l|}{0.025} & \multicolumn{2}{|l|}{0.0125} \\
\hline & $e_{\epsilon_{1}}^{n}$ & $e_{\epsilon_{2}}^{n}$ & $e_{\epsilon_{1}}^{n}$ & $e_{\epsilon_{2}}^{n}$ & $e_{\epsilon_{1}}^{n}$ & $e_{\epsilon_{2}}^{n}$ & $e_{\epsilon_{1}}^{n}$ & $e_{\epsilon_{2}}^{n}$ \\
\hline 2 & $2.1201 \mathrm{e}-2$ & $3.9697 e-2$ & $5.4220 \mathrm{e}-3$ & $1.0131 \mathrm{e}-2$ & $1.3673 e-3$ & $2.5533 e-3$ & $3.4332 \mathrm{e}-4$ & $6.4146 e-4$ \\
\hline 4 & $4.1031 e-2$ & $7.8091 \mathrm{e}-2$ & $1.0443 e-2$ & $1.9881 \mathrm{e}-2$ & $2.6281 e-3$ & $5.0027 e-3$ & $6.5986 e-4$ & $1.2555 \mathrm{e}-3$ \\
\hline 6 & $5.9886 \mathrm{e}-2$ & $1.1514 \mathrm{e}-1$ & $1.5225 \mathrm{e}-2$ & $2.9270 \mathrm{e}-2$ & $3.8276 \mathrm{e}-3$ & $7.3585 e-3$ & $9.6034 \mathrm{e}-4$ & $1.8457 \mathrm{e}-3$ \\
\hline 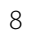 & $7.8493 e-2$ & $1.5154 \mathrm{e}-1$ & $1.9948 \mathrm{e}-2$ & $3.8497 e-2$ & $5.0129 e-3$ & $9.6738 e-3$ & $1.2572 \mathrm{e}-3$ & $2.4257 e-3$ \\
\hline 10 & $9.7041 \mathrm{e}-2$ & $1.8768 \mathrm{e}-1$ & $2.4657 e-2$ & $4.7665 e-2$ & $6.1945 \mathrm{e}-3$ & $1.1974 \mathrm{e}-2$ & $1.5533 e-3$ & $3.0020 \mathrm{e}-3$ \\
\hline
\end{tabular}

\begin{tabular}{|c|c|c|c|}
\hline$t$ & $E^{n}$ & $Q$ & $\tilde{E}$ \\
\hline 2 & 19.7989864026379 & 10.8827962014572 & 50.9215819473395 \\
\hline 4 & 19.7989864025761 & 10.8827962035498 & 50.9215813909925 \\
\hline 6 & 19.7989864025143 & 10.8827962044781 & 50.9215812185741 \\
\hline 8 & 19.7989864024516 & 10.8827962047988 & 50.9215811567766 \\
\hline 10 & 19.7989864023892 & 10.8827962049056 & 50.9215811324460 \\
\hline
\end{tabular}

advantages: Coupling with the matrix theory, we convert the proposed scheme into the vector difference one. The new scheme is high-accuracy which has the accuracy of $O\left(\tau^{2}+h^{4}\right)$; The new scheme is conservative and preserves the original conservative properties; The coefficient matrices of the scheme is symmetric and tridiagonal, so Thomas algorithm can be employed to solve it effectively. Some numerical results are reported to show the efficiency and accuracy of the scheme. 


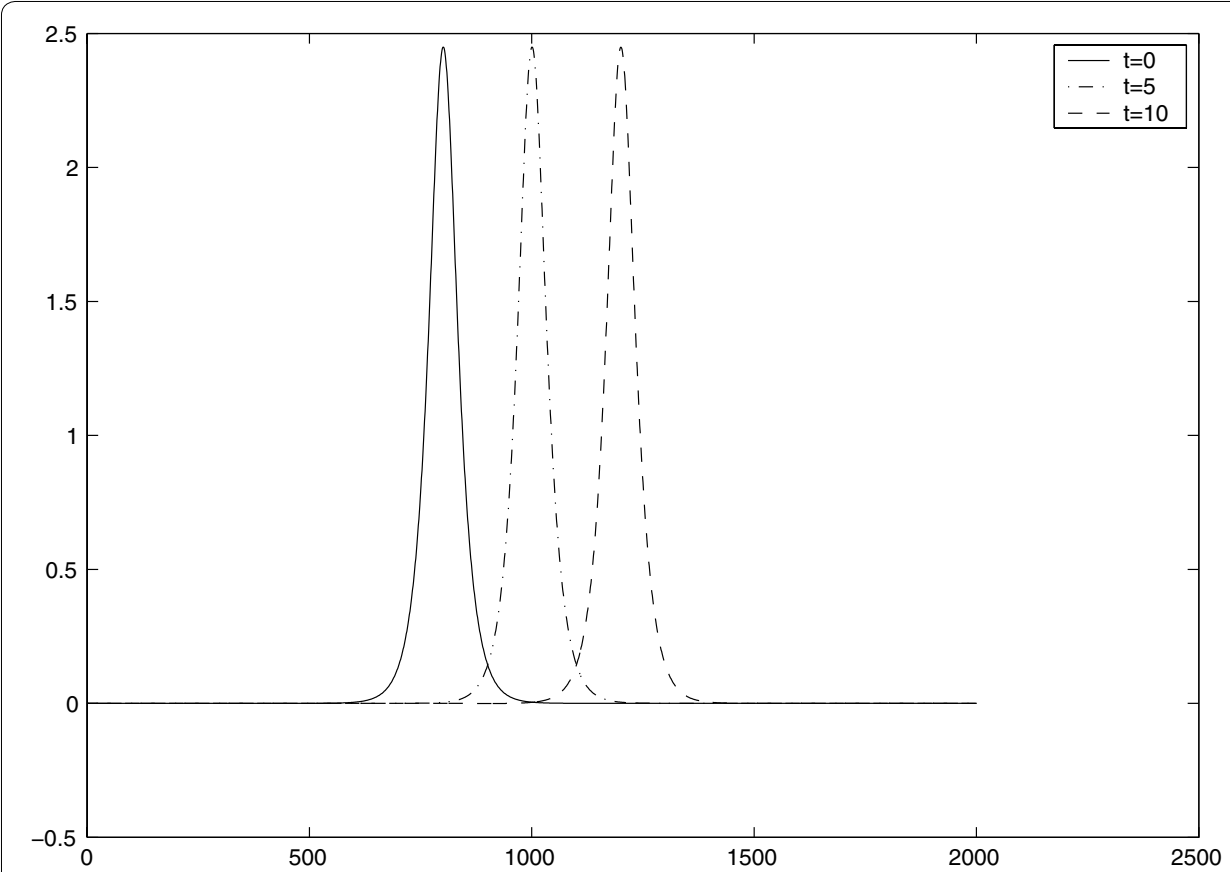

Fig. 3 Exact solutions $u(x, t)$ at $t=0$ and numerical solutions $u^{n}$ computed by the scheme (11)-(14) with $h=0.05, \tau=0.0025$ at $t=5$ and 10

Table 4 The maximal errors for numerical solutions $u^{n}$ of Scheme I and II at time $t=10$ with various $h$ and $\tau$

\begin{tabular}{lllcc}
\hline $\boldsymbol{( h , \boldsymbol { \tau } )}$ & $\mathbf{( 0 . 1 , 0 . 2 )}$ & $\mathbf{( 0 . 0 5 , 0 . 0 5 )}$ & $\mathbf{( 0 . 0 2 5 , 0 . 0 1 2 5 )}$ & $\mathbf{( 0 . 0 1 2 5 , \mathbf { 0 . 0 0 3 1 2 5 } )}$ \\
\hline Scheme I & $3.11496 \mathrm{e}-2$ & $1.53986 \mathrm{e}-3$ & $8.84019 \mathrm{e}-5$ & $5.40468 \mathrm{e}-6$ \\
CPU time (s) & 0.929105 & 2.412442 & 19.031491 & 255.135799 \\
Scheme II & $2.91603 \mathrm{e}-2$ & $1.87511 \mathrm{e}-3$ & $1.17545 \mathrm{e}-4$ & $7.35116 \mathrm{e}-6$ \\
CPU time (s) & 0.727532 & 2.229997 & 17.104052 & 239.567755 \\
\hline
\end{tabular}

\section{Authors' contributions}

The article was carried out in collaboration between all authors. Both authors have contributed to, read and approved the final manuscript.

\section{Author details}

${ }^{1}$ School of Mathematics and Information Science, Weifang University, Weifang 261061, China. ${ }^{2}$ School of Management, Qufu Normal University, Rizhao 276800, China. ${ }^{3}$ Department of Mathematics, Nanjing University of Aeronautics and Astronautics, Nanjing 210016, China.

\section{Acknowledgements}

This work is supported by the Natural Science Foundation of China (Nos. 11201343, 11401438), Natural Science Foundation of Shandong Province (ZR2012AM017, ZR2013FL032), A Project of Shandong Province Higher Educational Science and Technology Program (No. J14L152), the Youth Research Foundation of WFU (No. 2013Z11) and the Project of Science and Technology Program of Weifang (Grant No. 201301006).

\section{Competing interests}

Both authors declare that they have no competing interests.

Received: 17 February 2016 Accepted: 31 March 2016

Published online: 18 April 2016 


\section{References}

Akbari R, Mokhtari R (2014) A new compact finite difference method for solving the generalized long wave equation. Numer Func Anal Opt 35(2):133-152

Avilez-Valente P, Seabra-Santos FJ (2004) A Petrov-Galerkin finite element scheme for the regularized long wave equation. Comput Mech 34:256-270

Benjamin TB, Bona JL, Mahony JJ (1972) Model equations for long waves in nonlinear dispersive systems. Philos Trans R Soc Lond A 272:47-78

Cai J (2009) Multisymplectic numerical method for the regularized long-wave equation. Comput Phys Commun 180:1821-1831

Cai J (2010) A multisymplectic explicit scheme for the modified regularized long-wave equation. J Comput Appl Math 234:899-905

Cai J (2011) Some linearly and non-linearly implicit schemes for the numerical solutions of the regularized long-wave equation. Appl Math Comput 217:9948-9955

Dag I (2000) Least squares quadratic B-spline finite element method for the regularized long wave equation. Comput Methods Appl Mech Eng 182:205-215

Dag I, Ozer MN (2001) Approximation of the RLW equation by the least square cubic B-spline finite element method. Appl Math Model 25:221-231

Dag I, Saka B, Irk D (2004) Application of cubic B-splines for numerical solution of the RLW equation. Appl Math Comput 159:373-389

Dehghan M, Mohebbi A, Asgari Z (2009) Fourth-order compact solution of the nonlinear Klein-Gordon equation. Numer Algorithms 52:523-540

Esen A, Kutluay S (2006) Application of a lumped Galerkin method to the regularized long wave equation. Appl Math Comput 174(2):833-845

Fazal-i-Haq, Siraj-ul-Islam, Tirmizi IA (2010) A numerical technique for solution of the MRLW equation using quartic B-splines. Appl Math Model 34:4151-4160

Gao F, Qiao F, Rui H (2015) Numerical simulation of the modified regularized long wave equation by split least-squares mixed finite element method. Math Comput Simul 109:64-73

Gardner LRT, Gardner GA, Ayoub FA, Amein NK (1997) Approximations of solitary waves of the MRLW equation by B-spline finite element. Arab J Sci Eng 22:183-193

Gu H, Chen N (2008) Least-squares mixed finite element methods for the RLW equations. Numer Methods Partial Differ Eq. 24:749-758

Guo L, Chen H (2006) H1-Galerkin mixed finite element method for the regularized long wave equation. Computing 77:205-221

Khalifa A, Raslan K, Alzubaidi H (2007) A finite difference scheme for the MRLW and solitary wave interactions. Appl Math Comput 189:346-354

Khalifa A, Raslan K, Alzubaidi H (2008a) Numerical study using ADM for the modified regularized long wave equation. Appl Math Model 32:2962-2972

Khalifa A, Raslan K, Alzubaidi H (2008b) A collocation method with cubic B-spline for solving the MRLW eqution. J Comput Appl Math 212:406-418

Kutluay S, Esen A (2006) A finite difference solution of the regularized long wave equation. Math Probl Eng 2006:1-14

Labidi M, Omrani K (2011) Numerical simulation of the modified regularized long wave equation by He's variational iteration method. Numer Methods Partial Differ Eq. 27:478-489

Mokhtari Reza, Mohammadi Maryam (2010) Numerical solution of GRLW equation using Sinc-collocation method. Comput Phys Commun 181:1266-1274

Naz R, Khan MD, Naeem I (2013) Conservation laws and exact solutions of a class of nonlinear regularized long wave equations via double reduction theory and Lie symmetries. Commun Nonlinear Sci Numer Simul 18:826-834

Peregrine D (1996) Calculation of the developmentof undular bore. J Fluid Mech 25:321-330

Raslan KR (2009) Numerical study of the modified regularized long wave (MRLW) equation. Chaos Solitons Fractals 42:1845-1853

Saka B, Dag I (2008) A numerical solution of the RLW equation by Galerkin method using quartic B-splines. Commun Numer Methods Eng 24:1339-1361

Soliman AA, Raslan KR (2001) Collocation method using quadratic B-spline for the RLW equation. Int J Comput Math 78:399-412

Soliman AA, Hussien MH (2005) Collocation solution for RLW equation with septic spline. Appl Math Comput 161:623-636

Wang T (2014) Optimal point-wise error estimate of a compact difference scheme for the Klein-Gordon-Schrödinger equation. J Math Anal Appl 412:155-167

Wang T (2015) Uniform point-wise error estimates of semi-implicit compact finite difference methods for the nonlinear Schrödinger equation perturbed by wave operator. J Math Anal Appl 422:286-308

Wang T, Guo B (2011) Unconditional convergence of two conservative compact difference schemes for non-linear Schrödinger equation in one dimension. Sci Sin Math 41(3):207-233 (in Chinese)

Xie S, Li G, Yi S (2009) Compact finite difference schemes with high accuracy for one-dimensional nonlinear Schrödinger equation. Comput Methods Appl Mech Eng 198:1052-1060

Yan F, Liu H, Liu Z (2012) The bifurcation and exact travelling wave solutions for the modified Benjamin-Bona-Mahoney (mBBM) equation. Commun Nonlinear Sci Numer Simul 17:2824-2832

Zhang X (2004) Matrix analysis and applications. Tsinghua University Press, Beijing

Zhang L (2005) A finite difference scheme for the generalized regularized long-wave equation. Appl Math Comput 168:962-972

Zhou Y (1990) Application of discrete functional analysis to the finite difference method. Inter. Acad., Beijing 